\title{
A Study on the Convergence of Series Solution of Non-Newtonian Third Grade Fluid with Variable Viscosity: By Means of Homotopy Analysis Method
}

\author{
R. Ellahi, ${ }^{1}$ \\ ${ }^{1}$ Department of Mechanical Engineering, University of California Riverside, Bourns Hall, A373, \\ Riverside, CA 92521, USA \\ ${ }^{2}$ Department of Mathematics \& Statistics, FBAS, IIU, H-10, Islamabad 44000, Pakistan
}

Correspondence should be addressed to R. Ellahi, rellahi@engr.ucr.edu

Received 14 December 2011; Accepted 27 January 2012

Academic Editor: Teoman Özer

Copyright (c) 2012 R. Ellahi. This is an open access article distributed under the Creative Commons Attribution License, which permits unrestricted use, distribution, and reproduction in any medium, provided the original work is properly cited.

\begin{abstract}
This work is concerned with the series solutions for the flow of third-grade non-Newtonian fluid with variable viscosity. Due to the nonlinear, coupled, and highly complicated nature of partial differential equations, finding an analytical solution is not an easy task. The homotopy analysis method (HAM) is employed for the presentation of series solutions. The HAM is accepted as an elegant tool for effective solutions for complicated nonlinear problems. The solutions of (Hayat et al., 2007) are developed, and their convergence has been discussed explicitly for two different models, namely, constant and variable viscosity. An error analysis is also described. In addition, the obtained results are illustrated graphically to depict the convergence region. The physical features of the pertinent parameters are presented in the form of numerical tables.
\end{abstract}

\section{Introduction}

During the last few years, there has been substantial progress in the steady and unsteady flows of non-Newtonian fluids. A huge amount of literature is now available on the topic (see some studies [1-6]). All real fluids are diverse in nature. Hence in view of rheological characteristics, all non-Newtonian fluids cannot be explained by employing one constitutive equation. This is the striking difference between viscous and the non-Newtonian fluids. The rheological parameters appearing in the constitutive equations lead to a higher-order and complicated governing equations than the Navier-Stokes equations. The simplest subclass of differential-type fluids is called the second grade. In steady flow such fluids can predict the normal stress and does not show shear thinning and shear thickening behaviors. The thirdgrade fluid puts forward the explanation of shear thinning and shear thickening properties. 
Therefore, the present paper aims to study the pipe flow of a third-grade fluid. Some progress on the topic is mentioned in the studies $[7,8]$ and many references therein. In all these studies, variable viscosity is used. Massoudi and Christie [9] numerically examined the pipe flow of a third-grade fluid when viscosity depends upon temperature. Hayat et al. [10] presented the homotopy solution of the problem considered in [10] up to second-order deformation.

In this paper, the motivation comes from a desire to understand the convergence of the problem discussed in [10]. The relevant equations for flow and temperature have been solved analytically by using homotopy analysis method [11-15]. Here the convergence of the obtained solutions is explicitly shown, and that was not previously given in [10].

\section{Problem}

From [10], we have the equations (2.1) to (3.4) in nondimensional and nonlinear coupled partial differential equations of the form

$$
\begin{gathered}
\frac{1}{r} \frac{d}{d r}\left(r \mu(r)\left(\frac{d v}{d r}\right)\right)+\frac{\Lambda}{r} \frac{d}{d r}\left(r\left(\frac{d v}{d r}\right)^{3}\right)=c \\
\frac{d^{2} \theta}{d r^{2}}+\frac{1}{r}\left(\frac{d \theta}{d r}\right)+\Gamma\left(\frac{d v}{d r}\right)^{2}\left(\mu(r)+\Lambda\left(\frac{d v}{d r}\right)^{2}\right)=0
\end{gathered}
$$

subject to boundary conditions

$$
\begin{array}{ll}
v(1)=\theta(1)=0, & \frac{d v(0)}{d r}=\frac{d \theta(0)}{d r}=0, \\
v(1)=\theta(1)=0, & \frac{d v}{d r}(0)=\frac{d \theta}{d r}(0)=0 .
\end{array}
$$

\section{Solution of the Problem}

Our interest is to carry out the analysis for the homotopy solutions for two cases of viscosity, namely, constant and space-dependent viscous dissipation.

Case I. For constant viscosity model, we choose

$$
\mu=1
$$

For HAM solution, we select

$$
v_{0}(r)=\frac{c}{4}\left(r^{2}-1\right), \quad \theta_{0}=\frac{c^{2} \Gamma\left(1-r^{4}\right)}{64},
$$

as initial approximations of $v$ and $\theta$, respectively, which satisfy the linear operator and corresponding boundary conditions. We use the method of higher-order differential mapping [16] to choose the linear operator $\mathcal{L}$ which is defined by

$$
\left\llcorner_{1}=\frac{d^{2}}{d r^{2}}+\frac{1}{r} \frac{d}{d r},\right.
$$


such that

$$
\mathcal{L}_{1}\left(C_{1}+C_{2} \ln r\right)=0
$$

where $C_{1}$ and $C_{2}$ are the arbitrary constants.

If the convergence parameter is $\hbar$ and $0 \leq p \leq 1$ is an embedding parameter, then the zeroth-order problems become

$$
\begin{gathered}
(1-p) \mathcal{L}_{1}\left[v^{*}(r, p)-v_{0}(r)\right]=p \hbar \mathcal{N}_{1}\left[v^{*}(r, p), \theta^{*}(r, p)\right], \\
(1-p) \mathcal{L}_{1}\left[\theta^{*}(r, p)-\theta_{0}(r)\right]=p \hbar \mathcal{N}_{2}\left[v^{*}(r, p), \theta^{*}(r, p)\right], \\
v^{*}(1, p)=\theta^{*}(1, p)=0,\left.\quad \frac{\partial v^{*}(r, p)}{\partial r}\right|_{r=0}=\left.\frac{\partial \theta^{*}(r, p)}{\partial r}\right|_{r=0}=0,
\end{gathered}
$$

where the nonlinear parameters $\mathcal{N}_{1}$ and $\boldsymbol{N}_{2}$ are defined by

$$
\begin{aligned}
& \mathcal{N}_{1}\left[v^{*}(r, p), \theta^{*}(r, p)\right]=\frac{1}{r} \frac{d v^{*}}{d r}+\frac{d^{2} v^{*}}{d r^{2}}+\frac{\Lambda}{r}\left(\frac{d v^{*}}{d r}\right)^{3}+3 \Lambda\left(\frac{d v^{*}}{d r}\right)^{2} \frac{d^{2} v^{*}}{d r^{2}}-c, \\
& \mathcal{N}_{2}\left[v^{*}(r, p), \theta^{*}(r, p)\right]=\frac{1}{r} \frac{d \theta^{*}}{d r}+\frac{d^{2} \theta^{*}}{d r^{2}}+\Gamma\left(\frac{d v^{*}}{d r}\right)^{2}+\Gamma \Lambda\left(\frac{d v^{*}}{d r}\right)^{4} .
\end{aligned}
$$

For $p=0$ and $p=1$, we have

$$
v^{*}(r, 0)=v_{0}(r), \quad \theta^{*}(r, 0)=\theta_{0}(r), \quad v^{*}(r, 1)=v(r), \quad \theta^{*}(r, 1)=\theta(r)
$$

When $p$ increases from 0 to $1, v^{*}(r, p), \theta^{*}(r, p)$ vary from $v_{0}(r), \theta_{0}(r)$ to $v(r), \theta(r)$, respectively. By Taylor's theorem and (3.7), one can get

$$
v^{*}(r, p)=v_{0}(r)+\sum_{m=1}^{\infty} v_{m}(r) p^{m}, \quad \theta^{*}(r, p)=\theta_{0}(r)+\sum_{m=1}^{\infty} \theta_{m}(r) p^{m}
$$

where

$$
v_{m}(r)=\left.\frac{1}{m !} \frac{\partial^{m} v^{*}(r, p)}{\partial p^{m}}\right|_{p=0}, \quad \theta_{m}(r)=\left.\frac{1}{m !} \frac{\partial^{m} \theta^{*}(r, p)}{\partial p^{m}}\right|_{p=0}
$$

The convergence of the series (3.8) depends upon $\hbar$. We choose $\hbar$ in such a way that the series (3.8) is convergent at $p=1$; then, due to (3.7), we get

$$
v(r)=v_{0}(r)+\sum_{m=1}^{\infty} v_{m}(r), \quad \theta(r)=\theta_{0}(r)+\sum_{m=1}^{\infty} \theta_{m}(r)
$$


The $m$ th-order deformation problems are

$$
\begin{gathered}
\mathcal{L}_{1}\left[v_{m}(r)-\chi_{m} v_{m-1}(r)\right]=\hbar \Re 1_{m}(r), \\
\mathcal{L}_{1}\left[\theta_{m}(r)-\chi_{m} \theta_{m-1}(r)\right]=\hbar \Re 2_{m}(r), \\
v_{m}(1)=\theta_{m}(1)=0, \quad v_{m}^{\prime}(0)=\theta_{m}^{\prime}(0)=0,
\end{gathered}
$$

where the recurrence formulae $\mathfrak{R} 1$ and $\mathfrak{R} 2$ are given by

$$
\begin{aligned}
\mathfrak{R} 1_{m}(r)= & \frac{1}{r} \frac{d v_{m-1}}{d r}+\frac{d^{2} v_{m-1}}{d r^{2}}+\frac{\Lambda}{r} \sum_{k=0}^{m-1} \sum_{i=0}^{k}\left(\frac{d v_{m-1-k}}{d r}\right) \frac{d v_{k-i}}{d r} \frac{d v_{i}}{d r} \\
& +3 \Lambda \sum_{k=0}^{m-1} \sum_{i=0}^{k}\left(\frac{d v_{m-1-k}}{d r}\right) \frac{d v_{k-1}}{d r} \frac{d^{2} v_{i}}{d r^{2}}-\left(1-\chi_{m}\right) c \\
\mathfrak{R} 2_{m}(r)= & \frac{1}{r} \frac{d \theta_{m-1}}{d r}+\frac{d^{2} \theta_{m-1}}{d r^{2}}+\Gamma \sum_{k=0}^{m-1}\left(\frac{d v_{m-1-k}}{d r}\right) \frac{d v_{k}}{d r} \\
& +\Lambda \Gamma \sum_{k=0}^{m-1} \sum_{j=0}^{k} \sum_{i=0}^{j}\left(\frac{d v_{m-1-k}}{d r}\right) \frac{d v_{k-j}}{d r} \frac{d v_{j-i}}{d r} \frac{d v_{i}}{d r}
\end{aligned}
$$

in which

$$
X_{m}= \begin{cases}0, & m \leq 1 \\ 1, & m>1\end{cases}
$$

For constant viscosity, the velocity and temperature expressions up to second-order deformation are

$$
\begin{gathered}
v(r)=\frac{c}{4}\left(r^{2}-1\right)+\frac{h c^{3} \Lambda(2 h+3)\left(r^{4}-1\right)}{16}+\frac{h^{2} c^{5} \Lambda^{2}\left(r^{6}-1\right)}{32} \\
\theta(r)=\left[\begin{array}{c}
M_{1}\left(r^{4}-1\right)+M_{2}\left(r^{6}-1\right)+M_{3}\left(r^{8}-1\right)+M_{4}\left(r^{10}-1\right) \\
+M_{5}\left(r^{12}-1\right)+M_{6}\left(r^{14}-1\right)+M_{7}\left(r^{16}-1\right) \\
+M_{8}\left(r^{18}-1\right)+M_{9}\left(r^{20}-1\right)+M_{10}\left(r^{22}-1\right)
\end{array}\right] .
\end{gathered}
$$

Case II. For space-dependent viscosity, we take

$$
\mu=r
$$

For HAM solution, we select

$$
v_{0}(r)=\frac{c}{6}\left(r^{2}-1\right), \quad \theta_{0}=\frac{c^{4} \hbar^{4} \Gamma\left(1-r^{2}\right)}{64}
$$


As the initial approximation of $v$ and $\theta$. We select

$$
\left\llcorner_{2}=\frac{d^{2}}{d r^{2}}+\frac{2}{r} \frac{d}{d r}\right.
$$

such that

$$
\mathcal{L}_{2}\left(C_{3}+\frac{C_{4}}{r}\right)=0
$$

where $C_{3}$ and $C_{4}$ are arbitrary constants. The zeroth- and $m$ th-order deformation problems are

$$
\begin{gathered}
(1-p) \mathcal{L}_{2}\left[v^{*}(r, p)-v_{0}(r)\right]=p \hbar \mathcal{N}_{3}\left[v^{*}(r, p), \theta^{*}(r, p)\right], \\
(1-p) \mathcal{L}_{2}\left[\theta^{*}(r, p)-\theta_{0}(r)\right]=p \hbar \mathcal{N}_{4}\left[v^{*}(r, p), \theta^{*}(r, p)\right], \\
v^{*}(1, p)=\theta^{*}(1, p)=0,\left.\quad \frac{\partial v^{*}(r, p)}{\partial r}\right|_{r=0}=\left.\frac{\partial \theta^{*}(r, p)}{\partial r}\right|_{r=0}=0, \\
\mathcal{L}_{2}\left[v_{m}(r)-\chi_{m} v_{m-1}(r)\right]=\hbar \Re 3_{m}(r), \\
\mathcal{L}_{2}\left[\theta_{m}(r)-\chi_{m} \theta_{m-1}(r)\right]=\hbar \Re 4_{m}(r), \\
v_{m}(1)=\theta_{m}(1)=0, \quad v_{m}^{\prime}(0)=\theta_{m}^{\prime}(0)=0,
\end{gathered}
$$

where

$$
\begin{aligned}
& \mathcal{N}_{3}\left[v^{*}(r, p), \theta^{*}(r, p)\right]= \frac{2}{r} \frac{d v^{*}}{d r}+\frac{d^{2} v^{*}}{d r^{2}}+\frac{\Lambda}{r^{2}}\left(\frac{d v^{*}}{d r}\right)^{3}+\frac{3 \Lambda}{r}\left(\frac{d v^{*}}{d r}\right)^{2} \frac{d^{2} v^{*}}{d r^{2}}-\frac{c}{r} \\
& \mathcal{N}_{4}\left[v^{*}(r, p), \theta^{*}(r, p)\right]= \frac{1}{r} \frac{d \theta^{*}}{d r}+\frac{d^{2} \theta^{*}}{d r^{2}}+\Gamma\left(\frac{d v^{*}}{d r}\right)^{2}+\Gamma \Lambda\left(\frac{d v^{*}}{d r}\right)^{4}+\Gamma r\left(\frac{d v^{*}}{d r}\right)^{2}, \\
& \Re 3_{m}(r)= 2 r \frac{d v_{m-1}}{d r}+r^{2} \frac{d^{2} v_{m-1}}{d r^{2}}+\Lambda \sum_{k=0}^{m-1} \sum_{i=0}^{k}\left(\frac{d v_{m-1-k}}{d r}\right) \frac{d v_{k-i}}{d r} \frac{d v_{i}}{d r} \\
&+3 \Lambda r \sum_{k=0}^{m-1} \sum_{i=0}^{k}\left(\frac{d v_{m-1-k}}{d r}\right) \frac{d v_{k-i}}{d r} \frac{d^{2} v_{i}}{d r^{2}}-\left(1-\chi_{m}\right) c r \\
& \Re 4_{m}(r)=\frac{1}{r} \frac{d \theta_{m-1}}{d r}+\frac{d^{2} \theta_{m-1}}{d r^{2}}+\Gamma r \sum_{k=0}^{m-1}\left(\frac{d v_{m-1-k}}{d r}\right) \frac{d v_{k}}{d r} \\
&+\Lambda \Gamma \sum_{k=0}^{m-1} \sum_{j=0}^{k} \sum_{i=0}^{j}\left(\frac{d v_{m-1-k}}{d r}\right) \frac{d v_{k-j}}{d r} \frac{d v_{j-i}}{d r} \frac{d v_{i}}{d r} .
\end{aligned}
$$


For variable viscosity, the velocity and temperature expressions up to second-order deformation are

$$
\begin{gathered}
v(r)=\frac{h c}{2}(r-1)+\frac{c(2 h+3)\left(r^{2}-1\right)}{18}+\frac{c^{3} h \Lambda\left(r^{3}-1\right)}{81}, \\
\theta(r)=\left[M_{11}\left(r^{2}-1\right)+M_{12}\left(r^{3}-1\right)+M_{13}\left(r^{4}-1\right)+M_{14}\left(r^{5}-1\right)+M_{15}\left(r^{6}-1\right)\right],
\end{gathered}
$$

where the constant coefficients $M_{1}-M_{15}$ can be easily obtained through the routine calculation.

mth-order solutions

In both cases, for $p=0$ and $p=1$, we have

$$
\begin{array}{cc}
v^{*}(r ; 0)=v_{0}(r), & \theta^{*}(r ; 0)=\theta_{0}(y), \\
v^{*}(r ; 1)=v(r), & \theta^{*}(r ; 1)=\theta(r) .
\end{array}
$$

When $p$ increases from 0 to $1, v^{*}(r, p), \theta^{*}(r, p) \phi^{*}(r, p)$ varies from $v_{0}(r), \theta_{0}(r) \phi_{0}(r)$ to $v(r), \theta(r)$ and $\phi(r)$, respectively. By Taylor's theorem and (3.24) the general solutions can be written as

$$
v^{*}(r, p)=v_{0}(r)+\sum_{m=1}^{\infty} v_{m}(r) p^{m}, \quad \theta^{*}(r, p)=\theta_{0}(r)+\sum_{m=1}^{\infty} \theta_{m}(r) p^{m}
$$

where

$$
v_{m}(r)=\left.\frac{1}{m !} \frac{\partial^{m} v^{*}(r, p)}{\partial p^{m}}\right|_{p=0}, \quad \theta_{m}(r)=\left.\frac{1}{m !} \frac{\partial^{m} \theta^{*}(r, p)}{\partial p^{m}}\right|_{p=0}
$$

The convergence of (3.25) depends upon $\hbar$; therefore, we choose $\hbar$ in such a way that it should be convergent at $p=1$. In view of (3.24), finally the general form of $m$ th-order solutions is

$$
v(r)=v_{0}(r)+\sum_{m=1}^{\infty} v_{m}(r), \quad \theta(r)=\theta_{0}(r)+\sum_{m=1}^{\infty} \theta_{m}(r)
$$

\section{Discussion}

It is noticed that the explicit, analytical expressions (3.11), (19), (3.19), and (3.20) contain the auxiliary parameter $\hbar$. As pointed out by Liao [17], the convergence region and rate of approximations given by the HAM are strongly dependent upon $\hbar$. Figures 1 and 2 show the $\hbar$-curves of velocity and temperature profiles, respectively, just to find the range of $\hbar$ for the case of constant viscosity. The range for admissible values of $\hbar$ for velocity is $-2.4 \leq \hbar \leq 0.4$ and for temperature is $-2.2 \leq \hbar \leq 0.5$. Figures 4 and 5 represent the $\hbar$-curves for variable viscosity. The admissible ranges for both velocity and temperature profiles are $-3 \leq \hbar \leq$ 0.4 and $-2.8 \leq \hbar \leq 0.8$, respectively. In Figures 3 and 6 , the graphs of residual error are 


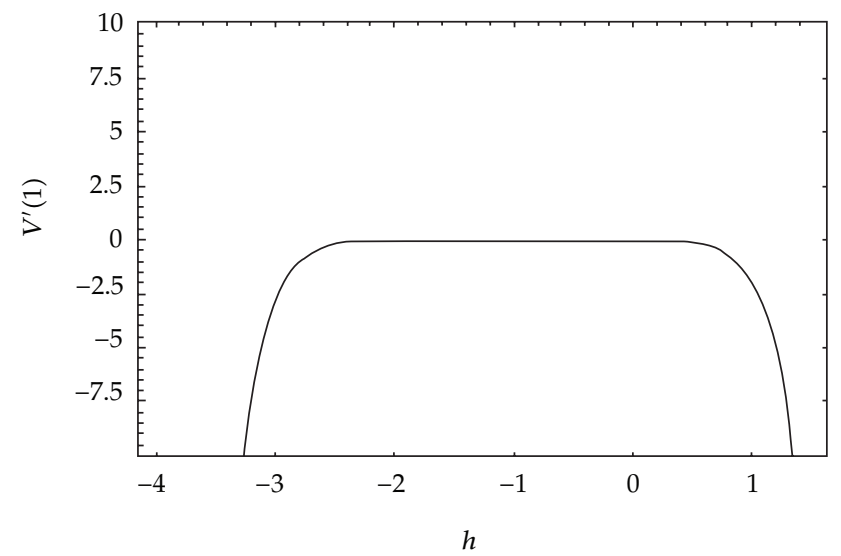

Figure 1: $\hbar$-curve for velocity in case of constant viscosity at 10th-order approximation.

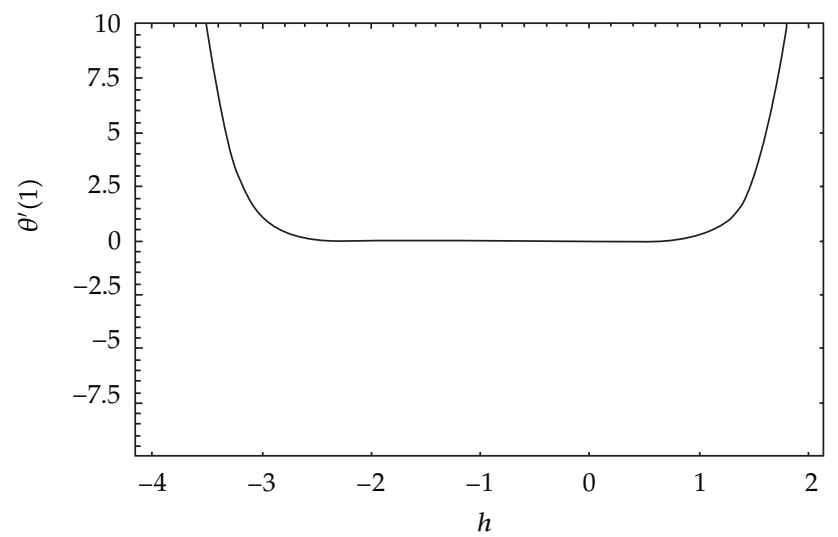

Figure 2: $\hbar$-curve for temperature in case of constant viscosity at 10th-order approximation.

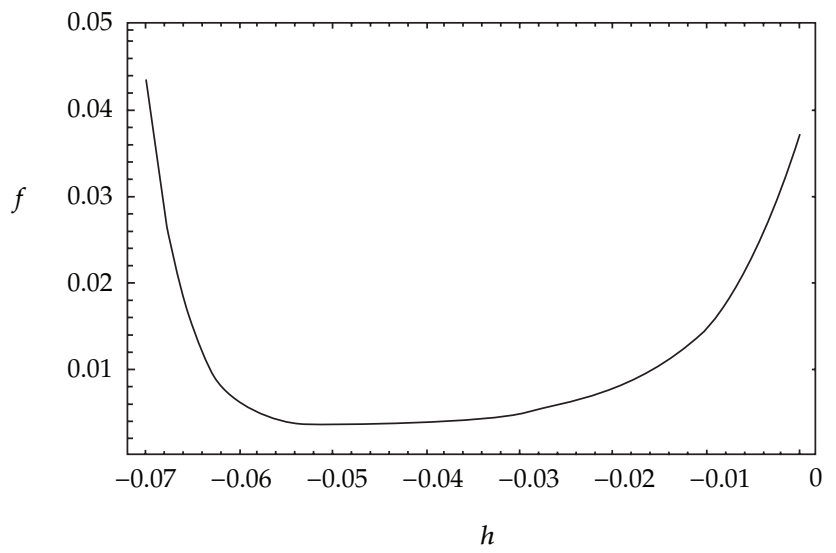

Figure 3: Residual error curve for constant viscosity. 


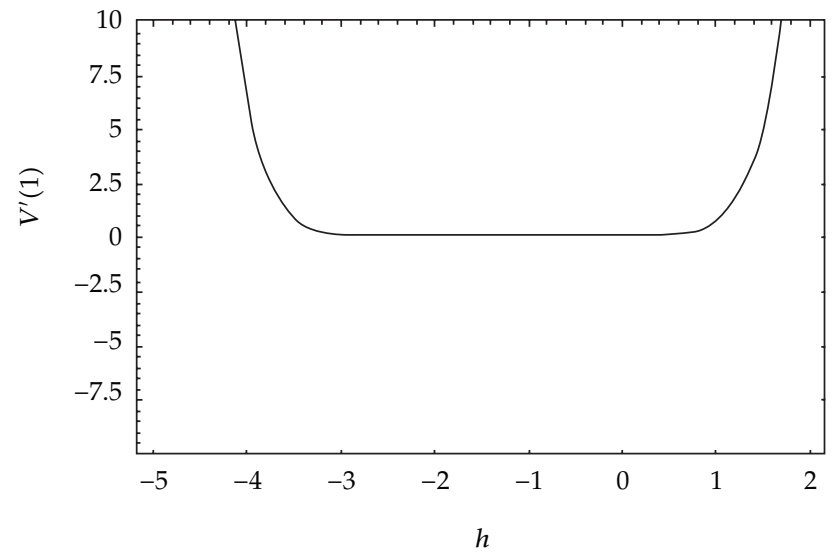

Figure 4: $\hbar$-curve for velocity in case of variable viscosity at 10th-order approximation.

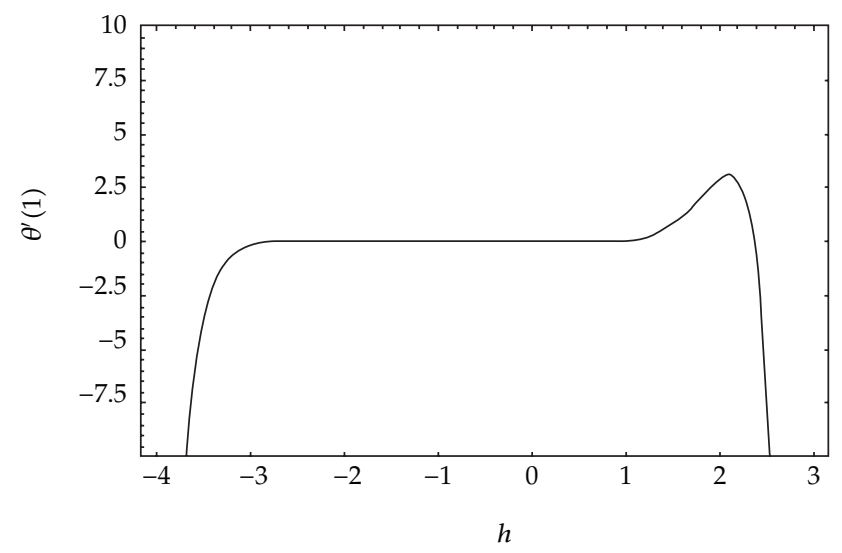

Figure 5: $\hbar$-curve for temperature in case of variable viscosity at 10th-order approximation.

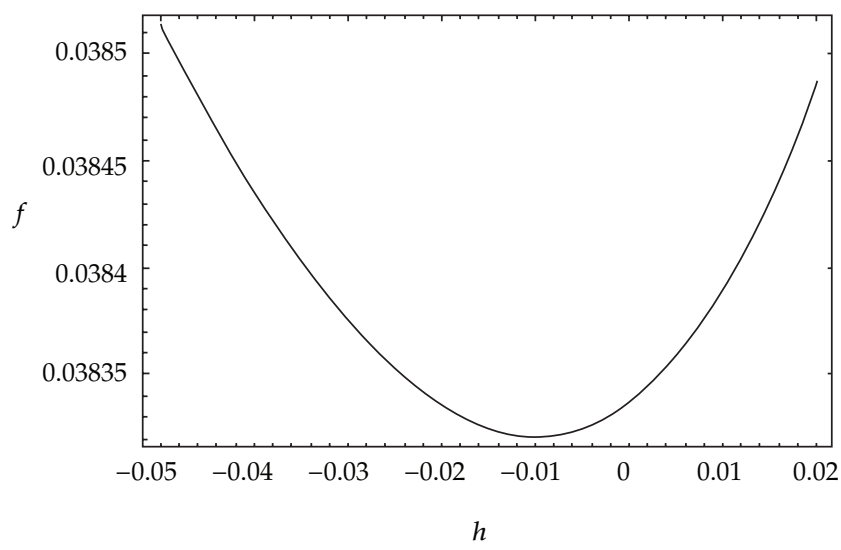

Figure 6: Residual error curve for variable viscosity. 
Table 1: Illustrating the variation of the velocity and temperature with $c$.

\begin{tabular}{lcccc}
\hline$h$ & $\Lambda$ & $c$ & $V$ & $\theta$ \\
\hline-0.01 & 1 & -1 & 1.673 & 0.006 \\
& & -2 & 3.191 & 0.068 \\
& & -3 & 4.4331 & 0.270 \\
& & -4 & 5.339 & 0.661 \\
& & -5 & 5.924 & 1.205 \\
\hline
\end{tabular}

Table 2: Illustrating the variation of the velocity and temperature with $\Lambda$.

\begin{tabular}{llcccc}
\hline$h$ & $c$ & $\Gamma$ & $\Lambda$ & $V$ & $\theta$ \\
\hline-0.01 & -1 & 1 & 0 & 1.700 & 0.243 \\
& & 5 & 1.571 & 2.002 \\
& & 10 & 1.455 & 3.209 \\
& & 15 & 1.353 & 4.011 \\
& & 20 & 1.263 & 4.520 \\
\hline
\end{tabular}

plotted for constant and variable viscosity, respectively. The error of norm 2 of two successive approximations over $[0,1]$ with HAM by 10th-order approximations is calculated by

$$
E_{2}=\sqrt{\frac{1}{11} \sum_{i=0}^{10}\left(v_{10}\left(\frac{i}{10}\right)\right)^{2}}=f . \quad \text { (say) }
$$

It is seen that the error is minimum at $\hbar=-0.01$. These values of $\hbar$ also lie in the admissible range of $\hbar$.

We use the widely applied symbolic computation software MATHEMATICA to see the effects of sundry parameters by Tables 1,2 , and 3 .

\section{Conclusion}

In this paper, the convergence of series solution for constant and variable viscosity in a thirdgrade fluid is presented. The steady pipe flow is considered. Convergence values and residual error are also examined in Figures 1 to 6 . To see the effects of emerging parameters for constant and variable viscosity, Tables 1 to 3 have been displayed. In Tables 1 and 2, it is found that the velocity and temperature increase with the decrease in pressure gradient and thirdgrade parameter, respectively, whereas Table 3 explains the variation of viscous dissipation parameter on velocity and temperature distributions. Here, it is revealed that the velocity and temperature decrease by increasing the viscous dissipation. It is observed that the results and figures [10] for important parameters $c, \Lambda$ and $\Gamma$ are correct and remain unchanged. 
Table 3: Illustrating the variation of temperature with $\Gamma$.

\begin{tabular}{|c|c|c|c|c|c|}
\hline$h$ & c & $\Lambda$ & $\Gamma$ & $V$ & $\theta$ \\
\hline \multirow[t]{6}{*}{-0.01} & 1 & 1 & & & \\
\hline & & & 0 & 0 & 0 \\
\hline & & & 5 & 0.075 & 3.242 \\
\hline & & & 10 & 0.158 & 6.484 \\
\hline & & & 15 & 0.249 & 9.726 \\
\hline & & & 20 & 0.351 & 12.969 \\
\hline
\end{tabular}

\section{Acknowledgments}

R. Ellahi thanks the United State Education Foundation Pakistan and CIES USA for honoring him by the Fulbright Scholar Award for the year 2011-2012. R. Ellahi is also grateful to the Higher Education Commission and PCST of Pakistan to award him the awards of NRPU and Productive Scientist, respectively.

\section{References}

[1] W. Tan and T. Masuoka, "Stability analysis of a Maxwell fluid in a porous medium heated from below," Physics Letters A, vol. 360, no. 3, pp. 454-460, 2007.

[2] T. Hayat and F. M. Mahomed, "Note on an exact solution for the pipe flow of a third-grade fluid," Acta Mechanica, vol. 190, no. 1-4, pp. 233-236, 2007.

[3] W. Tan and T. Masuoka, "Stokes' first problem for a second grade fluid in a porous half-space with heated boundary," International Journal of Non-Linear Mechanics, vol. 40, no. 4, pp. 515-522, 2005.

[4] M. Y. Malik, A. Hussain, and S. Nadeem, "Flow of a Jeffery-six constant fluid between coaxial cylinders with heat transfer analysis," Communications in Theoretical Physics, vol. 56, no. 2, pp. 345-351, 2011.

[5] M. Y. Malik, A. Hussain, S. Nadeem, and T. Hayat, "Flow of a third grade fluid between coaxial cylinders with variable viscosity," Zeitschrift fur Naturforschung A, vol. 64, no. 9-10, pp. 588-596, 2009.

[6] M. Hameed and S. Nadeem, "Unsteady MHD flow of a non-Newtonian fluid on a porous plate," Journal of Mathematical Analysis and Applications, vol. 325, no. 1, pp. 724-733, 2007.

[7] R. Ellahi, Steady and Unsteady Flow for Newtonian and Non-Newtonian Fluids: Basics, Concepts and Methods, VDM, Saarbrücken, Germany, 2009.

[8] R. Ellahi and S. Afzal, "Effects of variable viscosity in a third grade fluid with porous medium: an analytic solution," Communications in Nonlinear Science and Numerical Simulation, vol. 14, no. 5, pp. 2056-2072, 2009.

[9] M. Massoudi and I. Christie, "Effects of variable viscosity and viscous dissipation on the flow of a third grade fluid in a pipe," International Journal of Non-Linear Mechanics, vol. 30, no. 5, pp. 687-699, 1995.

[10] T. Hayat, R. Ellahi, and S. Asghar, "The influence of variable viscosity and viscous dissipation on the non-Newtonian flow: an analytical solution," Communications in Nonlinear Science and Numerical Simulation, vol. 12, no. 3, pp. 300-313, 2007.

[11] S. J. Liao, The proposed homotopy analysis technique for the solution of nonlinear problems, Ph.D. thesis, Shanghai Jiao Tong University, Shanghai, China, 1992.

[12] S. Liao, Beyond Perturbation: Introduction to Homotopy Analysis Method, vol. 2, Chapman \& Hall, Boca Raton, Fla, USA, 2004.

[13] S. Abbasbandy, "The application of homotopy analysis method to nonlinear equations arising in heat transfer," Physics Letters A, vol. 360, no. 1, pp. 109-113, 2006.

[14] R. Ellahi, "Effects of the slip boundary condition on non-Newtonian flows in a channel," Communications in Nonlinear Science and Numerical Simulation, vol. 14, no. 4, pp. 1377-1384, 2009.

[15] T. Hayat, R. Ellahi, P. D. Ariel, and S. Asghar, "Homotopy solution for the channel flow of a third grade fluid," Nonlinear Dynamics, vol. 45, no. 1-2, pp. 55-64, 2006. 
[16] R. A. Van Gorder and K. Vajravelu, "On the selection of auxiliary functions, operators, and convergence control parameters in the application of the homotopy analysis method to nonlinear differential equations: a general approach," Communications in Nonlinear Science and Numerical Simulation, vol. 14, no. 12, pp. 4078-4089, 2009.

[17] S.-J. Liao, "An analytic approximate technique for free oscillations of positively damped systems with algebraically decaying amplitude," International Journal of Non-Linear Mechanics, vol. 38, no. 8, pp. 1173-1183, 2003. 


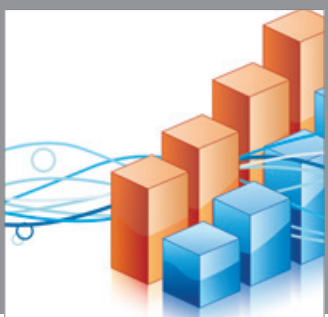

Advances in

Operations Research

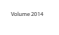

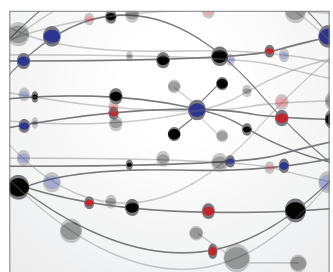

\section{The Scientific} World Journal
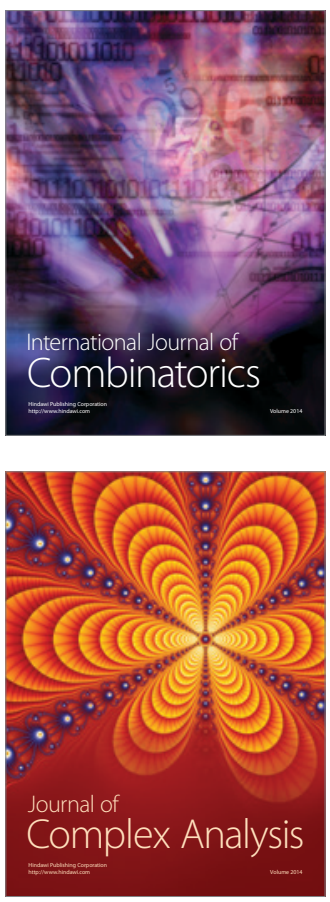

International Journal of

Mathematics and

Mathematical

Sciences
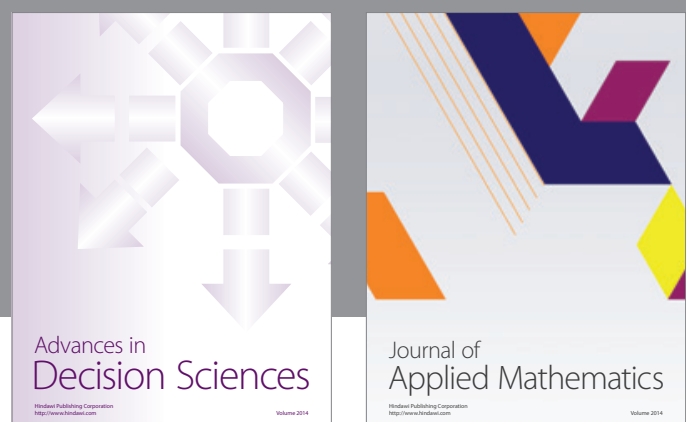

Journal of

Applied Mathematics
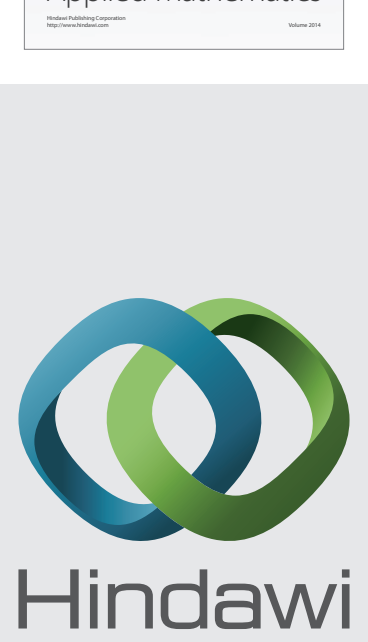

Submit your manuscripts at http://www.hindawi.com
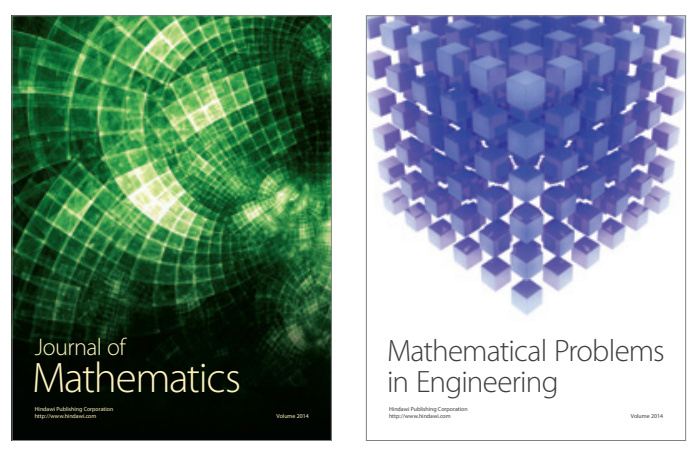

Mathematical Problems in Engineering
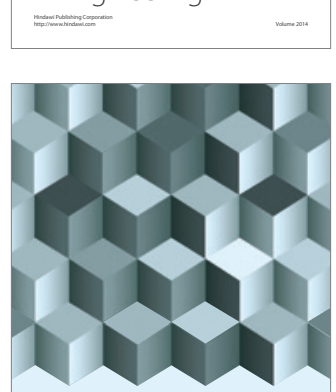

Journal of

Function Spaces
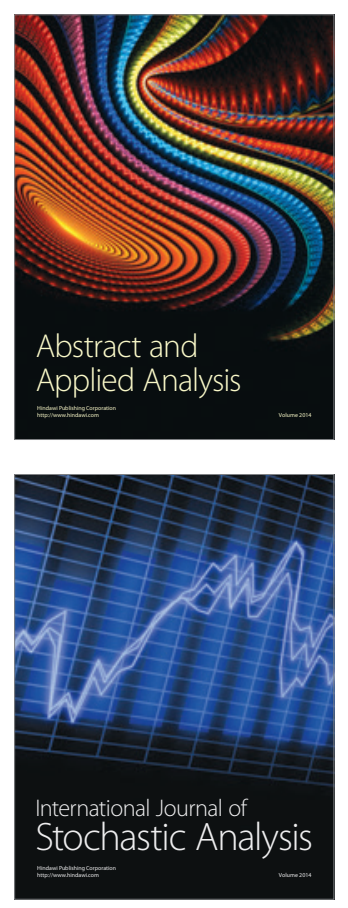

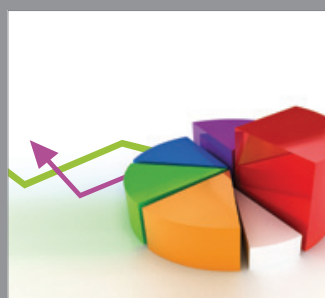

ournal of

Probability and Statistics

Promensencen
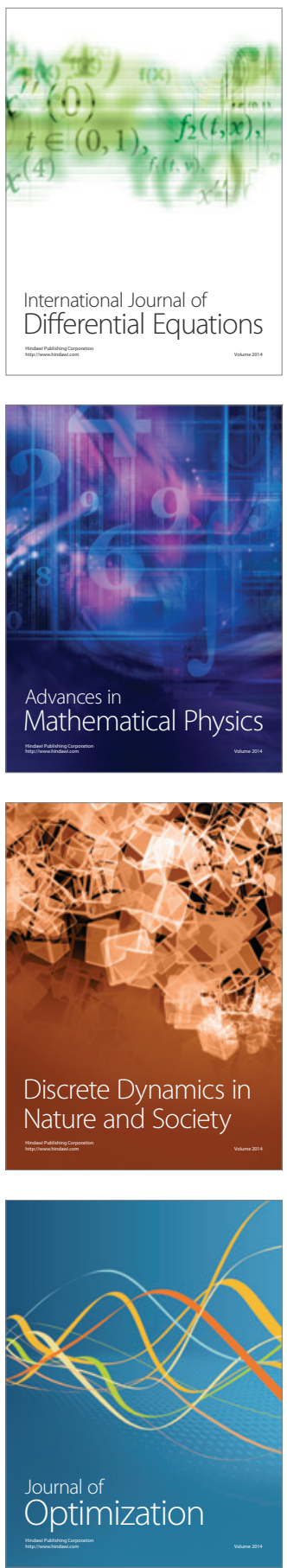